\title{
Adsorption isotherm studies of palm carotene extraction by synthetic polymer adsorbent
}

\begin{abstract}
The use of different synthetic polymer resin adsorbents, including HP 20, Exa 31, Exa 32 and Exa 50 for the removal of carotenes from crude palm oil was investigated. The adsorption of carotene was determined using several adsorption isotherm models such as Langmuir and Scatchard plots. The Langmuir adsorption model was found to be sufficient to describe the adsorption of carotenes by using these four types of resins, suggesting that the process was favorable, saturable and an equilibrated mechanism. A curve of Scatchard transformation plot showed that the adsorption involves multiple binding sites. The maximum uptake capacity of all resins used in isopropanol (IPA) was about 5 fold higher than the maximum uptake capacity obtained in n-hexane. The adsorbent found to be most efficient for carotene was HP 20 for experiment carried out in IPA. The carotenes concentration obtained by HP 20 in IPA was $31.44 \mathrm{mg} / \mathrm{L}$ compared to $7.12 \mathrm{mg} / \mathrm{L}$ obtained in $\mathrm{n}$-hexane.
\end{abstract}

Keyword: Carotene; Synthetic polymer; Adsorption 